\title{
An identity involving certain Hardy sums and Ramanujan's sum
}

Weiqiong Wang ${ }^{1,2}$ and Di Han ${ }^{2 *}$

*Correspondence: handi515@163.com

${ }^{2}$ Department of Mathematics, Northwest University, Xi'an, Shaanxi, P.R. China

Full list of author information is available at the end of the article

\section{Abstract}

The main purpose of this paper is using the properties of Gauss sums and the mean value theorem of the Dirichlet $L$-functions to study one kind of a hybrid mean value problem involving certain Hardy sums and Ramanujan's sum and to give an exact computational formula for it.

MSC: 11L05; 11L10

Keywords: Gauss sums; Ramanujan's sum; certain Hardy sums; hybrid mean value; computational formula

\section{Introduction}

Let $c$ be a natural number and $d$ be an integer prime to $c$. The classical Dedekind sums

$$
S(d, c)=\sum_{j=1}^{c}\left(\left(\frac{j}{c}\right)\right)\left(\left(\frac{d j}{c}\right)\right)
$$

where

$$
((x))= \begin{cases}x-[x]-\frac{1}{2}, & \text { if } x \text { is not an integer; } \\ 0, & \text { if } x \text { is an integer }\end{cases}
$$

describe the behavior of the logarithm of the eta-function (see [1] and [2]) under modular transformations. Funakura [3] gave an analogous transformation formula for the logarithm of the classical theta-function

$$
\theta(z)=\sum_{n=-\infty}^{+\infty} \exp \left(\pi i n^{2} z\right), \quad \operatorname{Im}(z)>0
$$

That is, put $V z=(a z+b)(c z+d)$ with $a, b, c, d \in Z, c>0$, and $a d-b c=1$. Then we have

$$
\log \theta(V z)=\log \theta(z)+\frac{1}{2} \log (c z+d)-\frac{1}{4} \pi i+\frac{1}{4} \pi i S_{1}(d, c)
$$

where $S_{1}(d, c)$ is defined as

$$
S_{1}(d, c)=\sum_{j=1}^{c-1}(-1)^{j+1+\left[\frac{d j}{c}\right]}
$$

\section{Springer}

( 2013 Wang and Han; licensee Springer. This is an Open Access article distributed under the terms of the Creative Commons Attribution License (http://creativecommons.org/licenses/by/2.0), which permits unrestricted use, distribution, and reproduction in any medium, provided the original work is properly cited. 
The sums $S_{1}(d, c)$ (and certain related ones) are sometimes called Hardy sums. They are closely connected with Dedekind sums. Regarding the properties of $S_{1}(d, c)$ and related sums, some authors studied them and obtained many interesting results; see [4-7] and [8]. For example, Wenpeng Zhang [7] proved the following conclusion: Let $p$ be an odd prime. Then, for any fixed positive integer $m$, we have the asymptotic formula

$$
\sum_{h=1}^{p-1}\left|S_{1}(h, p)\right|^{2 m}=p^{2 m} \cdot \frac{\zeta^{2}(2 m)\left(1-\frac{1}{4^{m}}\right)}{\zeta(4 m)\left(1+\frac{1}{4^{m}}\right)}+O\left(p^{2 m-1} \cdot \exp \left(\frac{6 \ln p}{\ln \ln p}\right)\right),
$$

where $\zeta(s)$ is the Riemann zeta-function and $\exp (y)=e^{y}$.

On the other hand, we define Ramanujan's sum $R_{q}(c)$ as follows (see Theorem 8.6 of [9]):

$$
R_{q}(c)=\sum_{\substack{k=1 \\(k, q)=1}}^{q} e^{\frac{2 \pi i k c}{q}}=\sum_{d \mid(c, q)} d \mu(q / d)
$$

where $\mu(n)$ is the famous Möbius function. Some related properties of $R_{q}(c)$ can also be found in $[9,10]$ and $[11]$.

The main purpose of this paper is using the properties of Gauss sums and the mean square value theorem of the Dirichlet $L$-functions to study a hybrid mean value problem involving certain Hardy sums and Ramanujan's sum and to give an exact computational formula for it. That is, we shall prove the following.

Theorem Let $q>1$ be an odd square-full number. Then we have the identity

$$
\sum_{\substack{h=1 \\(h, q)=1}}^{q} R_{q}(2 h+1) \cdot S_{1}(2 h, q)=\phi^{2}(q) \cdot \prod_{p \mid q}\left(1+\frac{1}{p}\right)
$$

where $\phi(q)$ denotes the Euler function, and $\prod_{p \mid q}$ denotes the product over all distinct prime divisors of $q$.

It is very interesting that the value is equal to zero in our theorem if we use $R_{q}(h+1)$ instead of $R_{q}(2 h+1)$.

For a general odd number $q \geq 3$, whether there exits a computational formula for

$$
\sum_{\substack{h=1 \\(h, q)=1}}^{q} R_{q}(2 h+1) \cdot S_{1}(2 h, q)
$$

is an open problem. Interested readers are welcome to study it with us.

\section{Several lemmas}

In this section, we shall give several lemmas, which are necessary in the proof of our theorem. Hereinafter, we shall use many properties of Gauss sums and character sums, all of which can be found in [9], so they will not be repeated here. First we have the following lemma. 
Lemma 1 Let $q>1$ be an odd number, and let $\chi$ be any non-principal character $\bmod q$. Then, for any integer $m$ with $(m, q)=1$, we have the identity

$$
\sum_{c=1}^{q} \chi(c) \cdot R_{q}(m c+1)=\bar{\chi}(m) \cdot \tau(\chi) \cdot \tau(\bar{\chi})
$$

where $\tau(\chi)=\sum_{a=1}^{q-1} \chi(a) e\left(\frac{a}{q}\right)$ denotes the classical Gauss sums, and $e(y)=e^{2 \pi i y}$.

Proof For any non-principal character $\chi \bmod q$, from the definition of $R_{q}(c)$ and the properties of Gauss sums, we have

$$
\begin{aligned}
\sum_{c=1}^{q} \chi(c) \cdot R_{q}(m c+1) & =\sum_{c=1}^{q} \sum_{\substack{b=1 \\
(b, q)=1}}^{q} \chi(c) e\left(\frac{b(m c+1)}{q}\right) \\
& =\sum_{\substack{b=1 \\
(b, q)=1}}^{q} e\left(\frac{b}{q}\right) \sum_{c=1}^{q} \chi(c) e\left(\frac{m b c}{q}\right) \\
& =\bar{\chi}(m) \sum_{\substack{b=1 \\
(b, q)=1}}^{q} \bar{\chi}(b) e\left(\frac{b}{q}\right) \sum_{c=1}^{q} \chi(m b c) e\left(\frac{m b c}{q}\right) \\
& =\bar{\chi}(m) \cdot \tau(\chi) \cdot \sum_{b=1}^{q} \bar{\chi}(b) e\left(\frac{b}{q}\right)=\bar{\chi}(m) \cdot \tau(\chi) \cdot \tau(\bar{\chi}) .
\end{aligned}
$$

This proves Lemma 1.

Lemma 2 Let $q>2$ be an integer. Then, for any integer a with $(a, q)=1$, we have the identity

$$
S(a, q)=\frac{1}{\pi^{2} q} \sum_{d \mid q} \frac{d^{2}}{\phi(d)} \sum_{\substack{\chi \bmod d \\ \chi(-1)=-1}} \chi(a)|L(1, \chi)|^{2},
$$

where $L(1, \chi)$ denotes the Dirichlet L-function corresponding to character $\chi \bmod d$.

Proof See Lemma 2 of [12].

Lemma 3 Let $q>0$ and $(h, q)=1$. Then we have the identity

$$
S_{1}(h, q)=-8 S(h+q, 2 q)+4 S(h, q) .
$$

Proof This formula is an immediate consequence of (5.9) and (5.10) in [6].

Lemma 4 Let $q>1$ be an odd number and $0<h<q$ with $(h, q)=1$. Then we have the identity

$$
S_{1}(2 h, q)=-20 \cdot S(2 h, q)+8 \cdot S(4 h, q)+8 \cdot S(h, q) .
$$


Proof From Lemma 2 and Lemma 3, we have

$$
\begin{aligned}
S_{1}(2 h, q)= & -8 \cdot S(2 h+q, 2 q)+4 \cdot S(2 h, q) \\
= & -\frac{4}{\pi^{2} q} \sum_{d \mid 2 q} \frac{d^{2}}{\phi(d)} \sum_{\substack{\chi \bmod d \\
\chi(-1)=-1}} \chi(2 h+q)|L(1, \chi)|^{2} \\
& +\frac{4}{\pi^{2} q} \sum_{d \mid q} \frac{d^{2}}{\phi(d)} \sum_{\chi \bmod d} \chi(2 h)|L(1, \chi)|^{2} \\
= & -\frac{4}{\pi^{2} q} \cdot \sum_{d \mid q} \frac{(2 d)^{2}}{\phi(2 d)=-1} \sum_{\chi \bmod 2 d} \chi(2 h+d)|L(1, \chi)|^{2} \\
= & -\frac{16}{\pi^{2} q} \cdot \sum_{d \mid q} \frac{d^{2}}{\phi(d)} \sum_{\substack{\chi \bmod d \\
\chi(-1)=-1}} \chi(2 h+q) \lambda(2 h+q)|L(1, \chi \lambda)|^{2} \\
= & -\frac{16}{\pi^{2} q} \cdot \sum_{d \mid q} \frac{d^{2}}{\phi(d)} \sum_{\substack{\chi \bmod d \\
\chi(-1)=-1}} \chi(2 h)|L(1, \chi \lambda)|^{2},
\end{aligned}
$$

where $\lambda$ is the principal character $\bmod 2$.

From the Euler infinite product formula, we have

$$
\begin{aligned}
|L(1, \chi \lambda)|^{2} & =\prod_{p_{1}}\left|1-\frac{\chi\left(p_{1}\right) \lambda\left(p_{1}\right)}{p_{1}}\right|^{-2}=\prod_{p_{1}>2}\left|1-\frac{\chi\left(p_{1}\right)}{p_{1}}\right|^{-2} \\
& =\left|1-\frac{\chi(2)}{2}\right|^{2} \cdot \prod_{p_{1}}\left|1-\frac{\chi\left(p_{1}\right)}{p_{1}}\right|^{-2}=\left(\frac{5}{4}-\frac{\chi(2)}{2}-\frac{\bar{\chi}(2)}{2}\right) \cdot|L(1, \chi)|^{2},
\end{aligned}
$$

where $\prod_{p_{1}}$ denotes the product over all primes $p_{1}$.

Now, combining (2.1), (2.2) and Lemma 2, we have the identity

$$
\begin{aligned}
S_{1}(2 h, q) & =-\frac{16}{\pi^{2} q} \cdot \sum_{d \mid q} \frac{d^{2}}{\phi(d)} \sum_{\substack{\chi \bmod d \\
\chi(-1)=-1}} \chi(2 h)|L(1, \chi \lambda)|^{2} \\
& =-\frac{16}{\pi^{2} q} \cdot \sum_{d \mid q} \frac{d^{2}}{\phi(d)} \sum_{\substack{\chi \bmod d \\
\chi(-1)=-1}} \chi(2 h)\left(\frac{5}{4}-\frac{\chi(2)}{2}-\frac{\bar{\chi}(2)}{2}\right) \cdot|L(1, \chi)|^{2} \\
& =-20 \cdot S(2 h, q)+8 \cdot S(4 h, q)+8 \cdot S(h, q) .
\end{aligned}
$$

This proves Lemma 4.

Lemma 5 Let $q>1$ be a square-full number (i.e., $q \geq 4$ and a prime $p \mid q$ implies $p^{2} \mid q$ ). Then, for any non-primitive character $\chi \bmod q$, we have the identity

$$
\sum_{a=1}^{q} \chi(a) e\left(\frac{a}{q}\right)=0 .
$$


Proof It is clear that from the multiplicative properties of Gauss sums we know that we only need to prove $q=p^{\alpha}$, a power of prime, where $\alpha \geq 2$. Suppose that $\chi$ is a nonprimitive character $\bmod p^{\alpha}$, then $\chi$ must be a character $\bmod p^{\alpha-1}$. So, from the definition of Gauss sums and the properties of a complete residue system $\bmod p^{\alpha}$ and trigonometric sums, we have

$$
\begin{aligned}
\sum_{a=1}^{p^{\alpha}} \chi(a) e\left(\frac{a}{p^{\alpha}}\right) & =\sum_{r=0}^{p-1} \sum_{a=1}^{p^{\alpha-1}} \chi\left(r p^{\alpha-1}+a\right) e\left(\frac{r p^{\alpha-1}+a}{p^{\alpha}}\right) \\
& =\sum_{a=1}^{p^{\alpha-1}} \chi(a) e\left(\frac{a}{p^{\alpha}}\right) \sum_{r=0}^{p-1} e\left(\frac{r}{p}\right)=0 .
\end{aligned}
$$

This proves Lemma 5.

Lemma 6 Let $q>3$ be an odd square-full number. Then we have the identities

$$
\begin{aligned}
& \sum_{\substack{\chi \bmod q \\
\chi(-1)=-1}}^{*}|L(1, \chi)|^{2}=\frac{\pi^{2}}{12} \cdot \frac{\phi^{3}(q)}{q^{2}} \prod_{p \mid q}\left(1+\frac{1}{p}\right) ; \\
& \sum_{\substack{\chi \bmod q \\
\chi(-1)=-1}}^{*} \chi(2) \cdot|L(1, \chi)|^{2}=\frac{\pi^{2}}{24} \cdot \frac{\phi^{3}(q)}{q^{2}} \prod_{p \mid q}\left(1+\frac{1}{p}\right),
\end{aligned}
$$

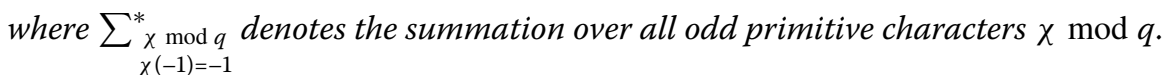

Proof From the definition of $S(a, q)$, we have the computational formula

$$
S(1, q)=\sum_{a=1}^{q-1}\left(\frac{a}{q}-\frac{1}{2}\right)^{2}=\frac{(q-1)(q-2)}{12 q} .
$$

From the reciprocity formula of $S(a, q)$, we know that for any positive integer $a$ with $(a, q)=$ 1 , we have the identity (see [4])

$$
S(a, q)+S(q, a)=\frac{q^{2}+a^{2}+1}{12 a q}-\frac{1}{4} .
$$

Applying this formula, we have

$$
S(2, q)=\frac{q^{2}+2^{2}+1}{24 q}-\frac{1}{4}-S(q, 2)=\frac{(q-1)(q-5)}{24 q} .
$$

From (2.3), Lemma 2 with $a=1$ and the Möbius inversion formula, we have

$$
\begin{aligned}
\frac{q^{2}}{\phi(q)} \sum_{\substack{\chi \bmod q \\
\chi(-1)=-1}}|L(1, \chi)|^{2} & =\pi^{2} \cdot \sum_{d \mid q} \mu(d) \cdot \frac{q}{d} \cdot S\left(1, \frac{q}{d}\right)=\pi^{2} \cdot \sum_{d \mid q} \mu(d) \cdot \frac{\left(\frac{q}{d}-1\right)\left(\frac{q}{d}-2\right)}{12} \\
& =\frac{\pi^{2}}{12} \cdot \phi(q) \cdot\left[q \cdot \prod_{p \mid q}\left(1+\frac{1}{p}\right)-3\right]
\end{aligned}
$$


or

$$
\sum_{\substack{\chi \bmod q \\ \chi(-1)=-1}}|L(1, \chi)|^{2}=\frac{\pi^{2}}{12} \cdot \frac{\phi^{2}(q)}{q^{2}} \cdot\left[q \cdot \prod_{p \mid q}\left(1+\frac{1}{p}\right)-3\right] .
$$

Then, using formula (2.5) and the Möbius inversion formula, we have

$$
\sum_{\substack{\chi \bmod q \\ \chi(-1)=-1}}^{*}|L(1, \chi)|^{2}=\sum_{d \mid q} \mu(d)\left(\sum_{\substack{\chi \bmod q / d \\ \chi(-1)=-1}}|L(1, \chi)|^{2}\right)=\frac{\pi^{2}}{12} \cdot \frac{\phi^{3}(q)}{q^{2}} \prod_{p \mid q}\left(1+\frac{1}{p}\right) .
$$

From (2.4), Lemma 2 with $a=2$ and the Möbius inversion formula, we also have

$$
\begin{aligned}
\frac{q^{2}}{\phi(q)} \sum_{\substack{\chi \bmod q \\
\chi(-1)=-1}} \chi(2) \cdot|L(1, \chi)|^{2} & =\pi^{2} \cdot \sum_{d \mid q} \mu(d) \cdot \frac{q}{d} \cdot S\left(2, \frac{q}{d}\right) \\
& =\pi^{2} \cdot \sum_{d \mid q} \mu(d) \cdot \frac{\left(\frac{q}{d}-1\right)\left(\frac{q}{d}-5\right)}{24} \\
& =\frac{\pi^{2}}{24} \cdot \phi(q) \cdot\left[q \cdot \prod_{p \mid q}\left(1+\frac{1}{p}\right)-6\right]
\end{aligned}
$$

or

$$
\sum_{\substack{\chi \bmod q \\ \chi(-1)=-1}} \chi(2) \cdot|L(1, \chi)|^{2}=\frac{\pi^{2}}{24} \cdot \frac{\phi^{2}(q)}{q^{2}} \cdot\left[q \cdot \prod_{p \mid q}\left(1+\frac{1}{p}\right)-6\right] .
$$

Then, using (2.7) and the Möbius inversion formula, we have

$$
\begin{aligned}
\sum_{\substack{\chi \bmod q \\
\chi(-1)=-1}}^{*} \chi(2) \cdot|L(1, \chi)|^{2} & =\sum_{d \mid q} \mu(d)\left(\sum_{\substack{\chi \bmod q / d \\
\chi(-1)=-1}} \chi(2) \cdot|L(1, \chi)|^{2}\right) \\
& =\frac{\pi^{2}}{24} \cdot \frac{\phi^{3}(q)}{q^{2}} \prod_{p \mid q}\left(1+\frac{1}{p}\right) .
\end{aligned}
$$

Now Lemma 6 follows from (2.6) and (2.8).

\section{Proof of the theorems}

In this section, we shall complete the proof of our theorem. Note that if $\chi$ is a primitive character $\bmod q$, then $|\tau(\chi)|=\sqrt{q}$, and $\tau(\chi) \cdot \tau(\bar{\chi})=\bar{\chi}(-1) \cdot \tau(\chi) \cdot \overline{\tau(\chi)}=\bar{\chi}(-1) \cdot q$. From Lemma 1, Lemma 2, Lemma 4, Lemma 5 and Lemma 6, we have

$$
\begin{aligned}
& \sum_{\substack{h=1 \\
(h, q)=1}}^{q} R_{q}(2 h+1) \cdot S_{1}(2 h, q) \\
& \quad=\sum_{\substack{h=1 \\
(h, q)=1}}^{q} R_{q}(2 h+1) \cdot(-20 \cdot S(2 h, q)+8 \cdot S(4 h, q)+8 \cdot S(h, q))
\end{aligned}
$$




$$
\begin{aligned}
& =-\frac{20}{\pi^{2} q} \cdot \sum_{d \mid q} \frac{d^{2}}{\phi(d)} \sum_{\substack{\chi \bmod d \\
\chi(-1)=-1}} \sum_{\substack{h=1 \\
(h, q)=1}}^{q} R_{q}(2 h+1) \cdot \chi(h) \cdot \chi(2) \cdot|L(1, \chi)|^{2} \\
& +\frac{8}{\pi^{2} q} \cdot \sum_{d \mid q} \frac{d^{2}}{\phi(d)} \sum_{\substack{\chi \bmod d \\
\chi(-1)=-1}} \sum_{\substack{h=1 \\
(h, q)=1}}^{q} R_{q}(2 h+1) \cdot \chi(h) \cdot \chi(4) \cdot|L(1, \chi)|^{2} \\
& +\frac{8}{\pi^{2} q} \cdot \sum_{d \mid q} \frac{d^{2}}{\phi(d)} \sum_{\substack{\chi \bmod d \\
\chi(-1)=-1}} \sum_{\substack{h=1 \\
(h, q)=1}}^{q} R_{q}(2 h+1) \cdot \chi(h) \cdot|L(1, \chi)|^{2} \\
& =-\frac{20}{\pi^{2} q} \cdot \sum_{d \mid q} \frac{d^{2}}{\phi(d)} \sum_{\substack{\chi \bmod d \\
\chi(-1)=-1}} \tau(\chi) \cdot \tau(\bar{\chi}) \cdot|L(1, \chi)|^{2} \\
& +\frac{8}{\pi^{2} q} \cdot \sum_{d \mid q} \frac{d^{2}}{\phi(d)} \sum_{\substack{\chi \bmod d \\
\chi(-1)=-1}} \tau(\chi) \cdot \tau(\bar{\chi}) \cdot \chi(2) \cdot|L(1, \chi)|^{2} \\
& +\frac{8}{\pi^{2} q} \cdot \sum_{d \mid q} \frac{d^{2}}{\phi(d)} \sum_{\substack{\chi \bmod d \\
\chi(-1)=-1}} \tau(\chi) \cdot \tau(\bar{\chi}) \cdot \bar{\chi}(2) \cdot|L(1, \chi)|^{2} \\
& =\frac{20}{\pi^{2}} \cdot \frac{q^{2}}{\phi(q)} \sum_{\substack{\chi \bmod q \\
\chi(-1)=-1}}^{*}|L(1, \chi)|^{2}-\frac{8}{\pi^{2}} \cdot \frac{q^{2}}{\phi(q)} \sum_{\substack{\chi \bmod q \\
\chi(-1)=-1}}^{*} \chi(2) \cdot|L(1, \chi)|^{2} \\
& -\frac{8}{\pi^{2}} \cdot \frac{q^{2}}{\phi(q)} \sum_{\substack{\chi \bmod q \\
\chi(-1)=-1}}^{*} \bar{\chi}(2) \cdot|L(1, \chi)|^{2} \\
& =\frac{20}{\pi^{2}} \cdot \frac{q^{2}}{\phi(q)} \cdot \frac{\pi^{2}}{12} \cdot \frac{\phi^{3}(q)}{q^{2}} \prod_{p \mid q}\left(1+\frac{1}{p}\right)-\frac{8}{\pi^{2}} \cdot \frac{q^{2}}{\phi(q)} \cdot \frac{\pi^{2}}{24} \cdot \frac{\phi^{3}(q)}{q^{2}} \prod_{p \mid q}\left(1+\frac{1}{p}\right) \\
& -\frac{8}{\pi^{2}} \cdot \frac{q^{2}}{\phi(q)} \cdot \frac{\pi^{2}}{24} \cdot \frac{\phi^{3}(q)}{q^{2}} \prod_{p \mid q}\left(1+\frac{1}{p}\right) \\
& =\phi^{2}(q) \cdot \prod_{p \mid q}\left(1+\frac{1}{p}\right),
\end{aligned}
$$

where we have used the identity

$$
\sum_{\substack{\chi \bmod q \\ \chi(-1)=-1}}^{*} \chi(2) \cdot|L(1, \chi)|^{2}=\sum_{\substack{\chi \bmod q \\ \chi(-1)=-1}}^{*} \bar{\chi}(2) \cdot|L(1, \chi)|^{2} .
$$

This completes the proof of our theorem.

\section{Competing interests}

The authors declare that they have no competing interests.

\section{Authors' contributions}

WW carried out the hybrid mean value problem involving certain Hardy sums and Ramanujan's sum and gave an exact computational formula. DH participated in the research and summary of the study. All authors read and approved the final manuscript. 


\section{Author details}

${ }^{1}$ School of Science, Chang'an University, Xi'an, Shaanxi, P.R. China. ${ }^{2}$ Department of Mathematics, Northwest University, Xi'an, Shaanxi, P.R. China.

\section{Acknowledgements}

The authors would like to thank the referees for their very helpful and detailed comments, which have significantly improved the presentation of this paper. This work is supported by the N.S.F. $(11071194,61202437)$ of P.R. China, and partly by the Fundamental Research Funds for the Central Universities of P.R. China (CHD2010JC101)

Received: 24 July 2013 Accepted: 9 August 2013 Published: 26 August 2013

\section{References}

1. Apostol, TM: Introduction to Analytic Number Theory. Springer, New York (1976)

2. Chowla, S: On Kloosterman's sums. Norske Vid. Selsk. Forh. 40, 70-72 (1967)

3. Funakura, T: On Kronecker's limit formula for Dirichlet series with periodic coefficients. Acta Arith. 55, 59-73 (1990)

4. Guy, RK: Unsolved Problems in Number Theory, 2nd edn. Springer, New York (1994)

5. Malyshev, AV: A generalization of Kloosterman sums and their estimates. Vestn. Leningr. Univ. 15, $59-75$ (1960) (in Russian)

6. $\mathrm{Xi}, \mathrm{P}, \mathrm{Yi}, \mathrm{Y}$ : On character sums over flat numbers. J. Number Theory 130, 1234-1240 (2010)

7. Zhang, W: On a problem of D. H. Lehmer and its generalization. Compos. Math. 86, 307-316 (1993)

8. Zhang, W: A problem of D. H. Lehmer and its mean square value formula. Jpn. J. Math. 29, 109-116 (2003)

9. Zhang, W: A problem of D. H. Lehmer and its generalization (II). Compos. Math. 91, 47-56 (1994)

10. Zhang, W: A mean value related to D. H. Lehmer's problem and the Ramanujan's sum. Glasg. Math. J. 54, 155-162 (2012)

11. Zhang, W: On the mean values of Dedekind sums. J. Théor. Nr. Bordx. 8, 429-442 (1996)

12. Zhang, W: On the difference between an integer and its inverse modulo $n$ (II). Sci. China Ser. A 46, 229-238 (2003)

doi:10.1186/1687-1847-2013-261

Cite this article as: Wang and Han: An identity involving certain Hardy sums and Ramanujan's sum. Advances in Difference Equations 2013 2013:261.

\section{Submit your manuscript to a SpringerOpen ${ }^{\circ}$ journal and benefit from:}

- Convenient online submission

- Rigorous peer review

Immediate publication on acceptance

- Open access: articles freely available online

- High visibility within the field

- Retaining the copyright to your article 